\title{
Le trouble \\ obsessionnel compulsif
}

João Flores Alves dos Santos ${ }^{1,2}$, Luc Mallet ${ }^{2,3}$

$>$ Le trouble obsessionnel compulsif (TOC) est une maladie fréquente et fréquemment comorbide. Le traitement habituel combine pharmacologie et psychothérapie, mais $30 \%$ des patients restent sévèrement symptomatiques, avec un retentissement fonctionnel important. Ces dernières années, l'intégration de données neuroanatomiques, neurochimiques, neuropsychologiques, génétiques et phénoménologiques a notamment permis d'identifier un dysfonctionnement cortico-sous-cortical. Ces avancées laissent entrevoir une meilleure compréhension de la physiopathologie et le développement de nouvelles thérapeutiques pour le TOC, dont la neuromodulation pour les formes sévères et résistantes de la maladie. <

\section{Définition du TOC}

Le trouble obsessionnel compulsif (TOC) est une maladie caractérisée par la présence d'idées obsédantes et de comportements compulsifs récurrents, ayant un impact négatif et parfois dévastateur sur le fonctionnement familial, social et professionnel de la personne atteinte.

Les obsessions sont des impulsions, des pensées ou des images intrusives, récurrentes et persistantes. Le sujet identifie ces obsessions comme le résultat de son activité mentale et critique leur caractère inapproprié. Les obsessions sont très souvent anxiogènes et elles engendrent une grande détresse chez le patient qui essaie infructueusement de les réprimer. La thématique des obsessions est très variée, allant de doutes permanents concernant des actes de la vie quotidienne (comme avoir bien fermé la porte, l'eau ou l'électricité), à la peur de perdre des objets, d'être contaminé, de faire du mal à autrui, d'être le responsable d'une catastrophe, etc.

Les compulsions sont des actes mentaux ou des comportements stéréotypés et répétitifs, ayant pour but de prévenir ou diminuer l'anxiété engendrée par les obsessions ou d'empêcher un événement redouté. Les com-

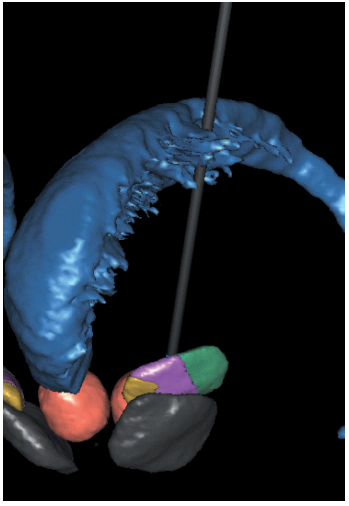

Service de psychiatrie de liaison et intervention crise, hôpitaux universitaires de Genève (HUG), Genève, Suisse ;

2 Équipe BEBG (behavior, emotion and basal ganglia), Institut du cerveau et de la moelle épinière (ICM), hôpital Pitié-Salpêtrière, 47, boulevard de l'Hôpital, 75013 Paris, France.

${ }^{3}$ Fondation FondaMental, Hôpital Albert Chenevier, 94000 Créteil, France. mail@jfasantos.net

pulsions ne visent pas le plaisir chez luc.mallet@upmc.fr Cependant, lorsqu'il essaie de réprimer ou de neutraliser ses compulsions, il voit son niveau d'anxiété s'aggraver. Les compulsions peuvent avoir un lien direct avec les obsessions, par exemple, se laver les mains de façon répétitive par peur d'être contaminé ou vérifier de façon répétitive si une porte ou un robinet sont bien fermés à la suite d'un doute permanent. Les compulsions peuvent aussi être une séquence d'actes stéréotypés, ritualisés et de constructions idiosyncratiques dont le sujet peine à expliquer leur raison.

\section{Épidémiologie et comorbidité}

Le TOC, qui a une prévalence élevée de 2-3\%, est considéré comme la 4 maladie par sa prévalence parmi les maladies psychiatriques $[1,2]$. La distribution selon l'âge est vraisemblablement bimodale, avec une apparition des symptômes dans l'enfance et une autre vers le début de l'âge adulte [3]. La distribution selon le genre est globalement homogène, cependant la survenue des symptômes lors de l'enfance est plus fréquente chez les garçons [3]. Les formes de début précoce semblent être associées à une plus grande sévérité et prévalence des symptômes, et aussi à une prévalence plus élevée de TOC chez les parents au premier degré [3, 4]. L'éventail de symptômes semblerait être plus large dans les formes de début précoce et serait plus fréquemment associé à un sentiment d'incomplétude, des expériences de just-right et des compulsions en absence d'obsession [4, 5]. Le TOC débutant dans l'enfance est également plus souvent associé à des tics $[3,4]$. Environ la moitié des sujets atteints d'un TOC souffrent d'une comorbidité psychiatrique : trouble dépressif, anxiété généralisée, agoraphobie ou trouble de panique et phobie sociale. L'abus ou la dépendance à l'alcool sont aussi plus fréquemment observés que 


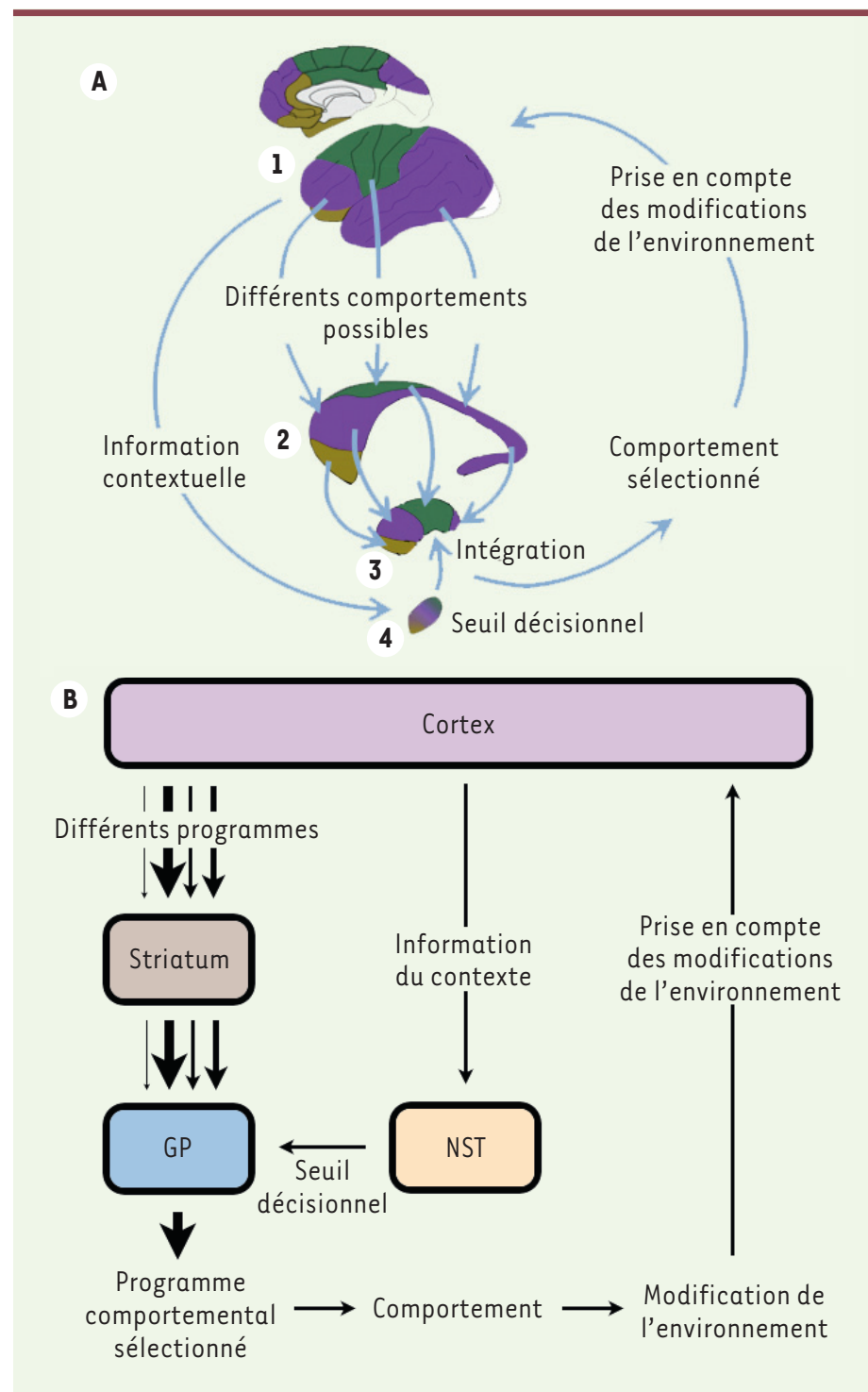

Figure 1. Le rôle du noyau sous-thalamique dans le TOC. Dans le processus cognitif de sélection d'une action, parmi les différentes possibilités alternatives (decision-making), le NST semble avoir une fonction de porte ou de seuil décisionnel des programmes moteurs en imposant la quantité d'information nécessaire provenant du cortex cérébral pour l'expression d'un comportement. 1 : cortex cérébral ; 2 : noyau caudé ; noyau globus pallidus (GP) ; noyau sousthalamique (NST).

dans la population générale [3]. La prévalence, l'âge de début et les comorbidités dépressives et anxieuses sont stables parmi différentes populations étudiées (États-Unis, Canada, Porto Rico, Allemagne, Taïwan, Corée du Sud et Nouvelle Zélande) [6]. Le contenu des idées obsédantes et des compulsions peut avoir une connotation culturelle, notamment religieuse. Toutefois, malgré la différence culturelle entre la population japonaise et la population occidentale, le contenu des symptômes obsessionnels et compulsifs est le même [7], ce qui renforce l'hypothèse d'un substrat organique commun.

\section{Le TOC au fil du temps}

Des descriptions de sujets souffrant d'une grande anxiété en lien avec des idées obsédantes et des comportements compulsifs remontent à plusieurs siècles, comme celles de l'Anatomie de la mélancolie de Robert Burton en 1621. Le terme obsession provient du latin obsessio et signifie «siège », «obsédé par le démon» dès le xve siècle. Le terme compulsion remonte au xIII ${ }^{e}$ siècle avec la signification de « contrainte». En 1838, Esquirol décrit ces cas en relevant le caractère « raisonnant » de la maladie, car l'individu maintient sa capacité critique, ainsi que ses facultés intellectuelles face à la «folie », la «monomanie raisonnante ». Après Legrand du Saulle avec La Folie du doute en 1875, et Pitres et Régis avec la Séméiologie des obsessions et des idées fixes en 1897, Pierre Janet contribue de façon décisive à la compréhension de la clinique avec la description d'un grand nombre de cas dans son ouvrage Les obsessions et la psychasthénie, publié en 1903. Il y défini la «psychasthénie » en tant que déficit de l'énergie mentale qui donne la place à des processus mentaux illogiques et irrationnels. II fait la séparation des symptômes en deux groupes: d'une part, les «idées obsédantes » et, d'autre part, les «agitations forcées ». Freud publie en 1894 Les Névropsychoses de défense avec la conceptualisation de la «névrose de contrainte» (zwang), traduit en français comme «névrose obsessionnelle ». Le modèle analytique de Freud considère la symptomatologie obsessionnelle et compulsive comme un produit des mécanismes de défense, le symptôme comme une formation réactionnelle, opposée au désir inconscient. Ce dernier est insupportable et donc réprimé, ce qui vise ainsi la protection et la conservation de l'intégrité du psychisme du sujet. Freud approfondira le sujet avec la monographie L'homme aux rats, en 1909.

La dénomination de «névrose obsessionnelle » se transforme au fil du temps avec l'évolution de la nosographie. Le DSM-I (first diagnostic and statistical manual) de 1952 fait état d'une catégorie de «trouble psychonévrotique, avec réaction obsessionnelle compulsive»; le DSM-II de 1968 de «névrose obsessionnelle compulsive»; et le DSM-III de 1980 de «trouble obsessionnel compulsif » parmi le groupe des «troubles anxieux », restant en tant que tel dans le DSM-IV-TR de 2000.

\section{Comprendre le TOC}

\section{Approche psychologique}

Parmi les modèles psychologiques contemporains du TOC, le modèle cognitivo-comportemental semble le plus opérationnel du point de vue de l'observation clinique et de l'expérimentation. Ce modèle formule 
I'hypothèse que les pensées intrusives sont, à la base, normales et fréquentes chez tout individu. Néanmoins, le sujet souffrant d'un TOC interprète (interprétation dysfonctionnelle) ces pensées comme de grande importance, inacceptables, immorales ou menaçantes. II s'ensuit une grande anxiété chez le sujet qui essaye de réprimer ces pensées à travers des compulsions. Les compulsions acquièrent un caractère persistant et excessif, car elles sont renforcées par l'anxiolyse immédiate et la disparition transitoire des pensées intrusives, et éloignent le sujet de la possibilité de corriger le dysfonctionnement d'interprétation initial [3].

\section{Approche biologique}

\section{Génétique}

Dans le TOC, l'héritabilité (degré d'influence probable des facteurs génétiques pour un phénotype spécifique dans une population donnée) est globalement estimée à 27-49\%, et à $65 \%$ chez les sujets dont les symptômes surviennent lors de l'enfance et l'adolescence. La variance restante est attribuée aux différents facteurs environnementaux [3]. Au sein des dimensions symptomatologiques, le facteur «accumulation » aurait l'héritabilité la plus élevée [3, 5]. Dans les études de jumeaux avec un TOC, des valeurs de concordance de $53 \%$ à $87 \%$ sont retrouvées pour les jumeaux monozygotes, et de $22 \%$ à $47 \%$ pour les jumeaux dizygotes $[3,8,9]$.

L'implication retrouvée de différents gènes évoque un modèle polygénique du TOC, lié aux systèmes sérotoninergique, glutamatergique, et dopaminergique. Plus récemment, des données génétiques impliquent également les systèmes cholinergique, opioïde endogène, GABA (acide $\gamma$-aminobutyrique) et substance $P[10,11]$.

\section{Neurochimie}

Les neurotransmetteurs possiblement impliqués dans la physiopathologie du TOC et les plus étudiés sont la sérotonine, la dopamine et le glutamate. L'observation d'une amélioration de la symptomatologie obsessionnelle-compulsive sous clomipramine, un antidépresseur tricyclique qui a comme principal mécanisme d'action l'inhibition de la recapture de sérotonine, constitue le premier élément en faveur de l'hypothèse d'un dysfonctionnement du système sérotoninergique dans le TOC [1]. Le transporteur de sérotonine est la principale cible de l'action des inhibiteurs de la recapture de la sérotonine (IRS) et une sensibilité augmentée du récepteur postsynaptique de sérotonine est retrouvée chez les patients souffrant d'un TOC [12]. Certains polymorphismes du gène codant pour le transporteur de sérotonine 5-HTT (= SLC6A4) et du gène codant pour le récepteur sérotoninergique postsynaptique 5HTR2A pourraient conférer une susceptibilité au développement de la symptomatologie du TOC $[1,3]$.

Des concentrations de glutamate élevées ont été retrouvées dans le liquide céphalorachidien chez des patients TOC [13]. Des études de spectroscopie par résonance magnétique nucléaire corroborent l'implication des anomalies du système glutamatergique dans la physiopathologie du TOC [14]. Enfin, comme évoqué plus haut, des études génétiques montrent une association du TOC avec les gènes SAPAP3
(SAP90-associated protein 3) et SLCIAI (solute carrier family 1, member 1) qui codent pour des transporteurs de glutamate $[8,9,15]$. II a été suggéré que l'effet thérapeutique observé avec les IRS pourrait être aussi associé à l'action modulatrice que ces médicaments exercent sur les concentrations glutamatergiques [11]. L'implication de la dopamine a été évoquée devant l'exacerbation des symptômes de TOC après administration d'agonistes dopaminergiques, et devant l'effet bénéfique consécutif à l'utilisation d'antagonistes dopaminergiques dans certaines formes de TOC réfractaire [1]. En outre, la recherche de polymorphismes de minisatellites (VNTR) du récepteur D4 de la dopamine montre une association avec le TOC $[16,17]$.

\section{PANDAS}

Les PANDAS (pediatric autoimmune neuropsychiatric disorders associated with streptococcal infections) désignent des tableaux pédiatriques avec une symptomatologie obsessionnelle-compulsive, avec ou sans tics, après infection par des streptocoques $\beta$-hémolytiques du groupe $A$, et une inflammation concomitante des ganglions de la base, avec une rémission complète ou quasi-complète des symptômes quand ils sont traités correctement avec des antibiotiques. L'hypothèse auto-immune découle de l'idée que l'infection initiale conduit à la production d'auto-anticorps qui réagissent avec les protéines des ganglions de la base [18]. Cependant, ces auto-anticorps n'ont pas été jusqu'à présent formellement retrouvés dans les PANDAS [19]. L'intérêt conceptuel des PANDAS est d'intégrer d'éventuels processus immunologiques dans la physiopathologie du TOC, notamment de l'immunité à médiation cellulaire, en faisant l'hypothèse d'un dysfonctionnement du système glutamatergique et de l'action modulatrice du glutamate sur les lymphocytes $T$ [11].

\section{Pathologie de réseau}

Une implication des ganglions de la base dans la physiopathologie du TOC a été évoquée suite à l'observation clinique de sujets souffrant de lésions focales des noyaux gris centraux, qui développent une symptomatologie de type obsessionnelle-compulsive, avec des caractéristiques neuropsychiatriques similaires à celles d'un TOC et des réponses favorables au même traitement que celui qui est utilisé dans le TOC [15].

Les travaux réalisés en imagerie cérébrale mettent en évidence des altérations anatomiques et fonctionnelles de parties spécifiques du cerveau des patients souffrant d'un TOC. Au niveau cortical, les principales anomalies 


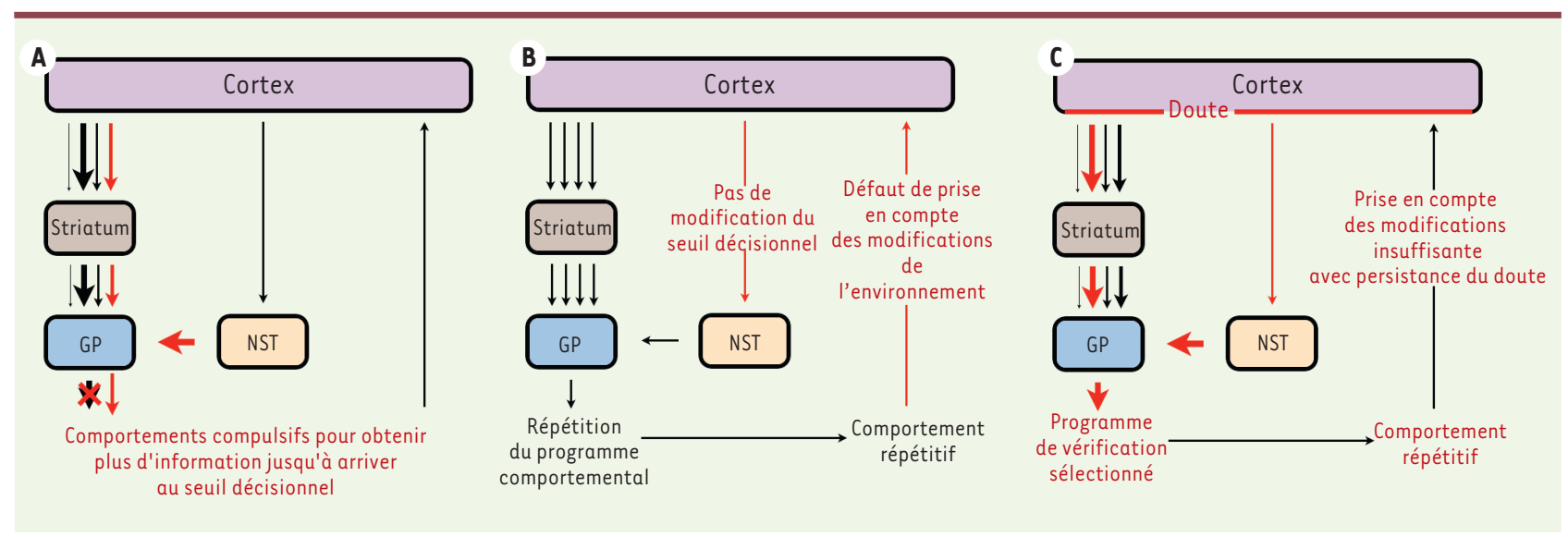

Figure 2. Modèles physiopathologiques pour le TOC. A. L'hyperactivité du NST est responsable d'un seuil décisionnel très élevé, engendrant des comportements compulsifs de vérification dans le but d'obtenir de l'environnement l'information nécessaire pour arriver au seuil. Les idées obsédantes sont ainsi une tentative de l'individu de rationaliser son comportement compulsif. B. Un dysfonctionnement de la prise en compte des modifications engendre des difficultés d'intégration du résultat comportemental. L'individu est ainsi empêché d'actualiser l'information contextuelle disponible et ne peut pas modifier le seuil décisionnel du NST. Le comportement exprimé est considéré comme la meilleure option, et il est donc répété. $\boldsymbol{C}$. Des dysfonctionnements corticaux sont responsables d'un doute permanent qui modifie le seuil décisionnel et qui permet uniquement l'expression de comportements apaisants de ce doute. NST : noyau sous-thalamique; GP : globus pallidus.

sont retrouvées pour le cortex orbitofrontal et le cortex cingulaire antérieur avec des différences volumétriques de la substance grise et de l'activité métabolique basale. II existe une corrélation entre les anomalies du cortex orbitofrontal et la sévérité des symptômes, avec une diminution de l'hyperactivité après un traitement efficace [20, 21]. Au niveau sous-cortical, des différences volumétriques de la substance grise sont retrouvées au niveau du thalamus et du noyau caudé, corrélées à la sévérité des symptômes et la normalisation de l'activité métabolique basale après traitement efficace [15, 20,22]. Ces modifications, retrouvées aux niveaux cortical et du système des ganglions de la base, évoquent un dysfonctionnement d'une boucle noyaux gris centraux-thalamo-cortical dont l'origine pourrait provenir du cortex orbitofrontal $[15,17,23]$. Des changements de la substance blanche sont aussi mis en évidence par l'imagerie en tenseur de diffusion, avec une corrélation positive entre la sévérité des symptômes et les valeurs d'anisotropie fractionnelle des fibres nerveuses, évoquant ainsi une «hyperconnectivité » dans le TOC [24]. L'association de l'augmentation de l'activité métabolique au niveau du cortex orbitofrontal du noyau caudé avec le TOC est très robuste ; elle est probablement un des résultats le plus reproduit dans les études d'imagerie cérébrale en psychiatrie (Figures 1 et 2).

L'expérimentation animale apporte également des données en faveur de l'implication des ganglions de la base dans la physiopathologie du TOC ou, tout au moins, celle des comportements d'allure compulsive. Chez des souris avec une délétion du gène SAPAP3 (exprimé au niveau du striatum), on observe un comportement de type anxieux avec un grooming compulsif qui s'améliore sous IRS [9]. De même, des micro-injections de bicuculline (antagoniste GABA) dans différentes parties du striatum et du pallidum externe de primates mettent en évidence le rôle de la partie ventromédiane (limbique) de ces noyaux dans des comportements d'allure compulsive $[25,26]$, alors que la partie antéromédiane du noyau sous-thalamique (NST) serait impliquée dans le contrôle du comportement [27].

\section{Traitement du TOC}

\section{Traitement pharmacologique}

L'utilisation des IRS est validée par une méta-analyse montrant une moyenne de taille d'effet élevée $(0,91)$ sur les symptômes du TOC [3, 28]. Malgré l'amélioration de la symptomatologie obsessionnelle-compulsive sous IRS, environ la moitié des sujets continuent de présenter des symptômes cliniquement significatifs. L'addition d'un antagoniste dopaminergique, comme la risperidone, peut améliorer les symptômes, surtout s'il existe des tics associés $[29,30]$.

Quelques études pharmacologiques préliminaires ont rapporté une diminution de l'intensité de la symptomatologie du TOC avec des antiglutamatergiques, notamment le riluzole et la mémantine $[3,11]$.

\section{Traitement psychothérapeutique}

La thérapie cognitivocomportementale (TCC) avec exposition et prévention de la réponse est le traitement psychothérapeutique le plus validé. Elle vise à apprendre au patient que son anxiété ne persiste pas indéfiniment et que les compulsions ne sont pas totalement indispensables pour éviter les dommages [31]. Le taux de réponse est de $83 \%$ avec une efficacité égale à 


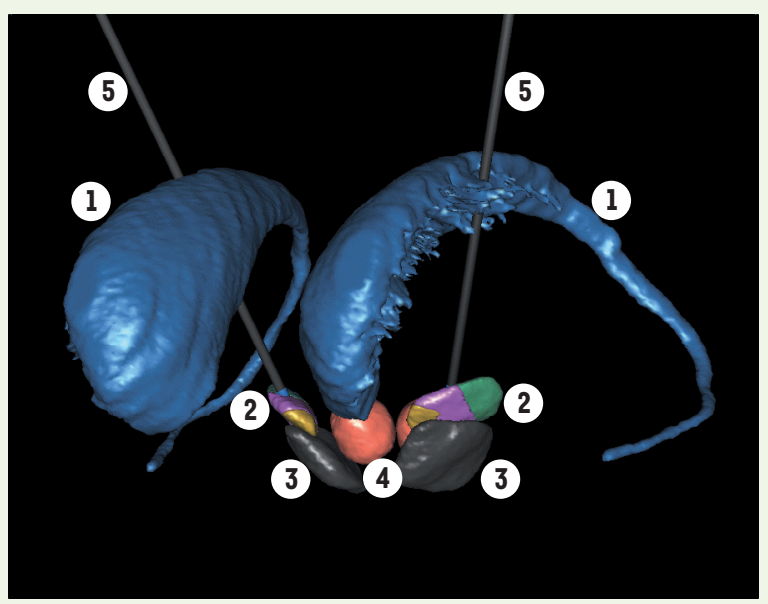

Figure 3. Stimulation cérébrale profonde du NST. Image 3D d'implantation d'électrodes bilatérale pour stimulation cérébrale profonde du NST. 1 : noyau caudé ; 2 : noyau sous-thalamique (territoires: moteur en vert; associatif en violet ; limbique en jaune) ; 3 : substance noire ; 4 : noyau rouge ; 5 : électrodes. Crédits : nous tenons à remercier Jérôme Yelnik, Eric Bardinet et Sara Fernandez Vidal pour l'image de l'atlas 3D histologique des GB.

celle d'une monothérapie pharmacologique par des IRS dans les traitements de première intention [32].

\section{Pharmacologie plus psychothérapie}

Le traitement habituel du TOC associe traitement pharmacologique et psychothérapeutique, bien que l'efficacité de cette combinaison en comparaison de la monothérapie n'ait pas été confirmée par méta-analyse [33]. Des données montrant que le récepteur NMDA ( $\mathrm{N}$-méthyl-D-aspartate) serait impliqué dans des processus d'extinction de la peur ont inspiré des essais cliniques récents avec thérapie combinée de D-cyclosérine (agoniste partiel du récepteur NMDA) et TCC, postulant un renforcement de l'extinction de la peur apprise dans le TOC. Les résultats préliminaires semblent prometteurs [34].

Toutefois, malgré la combinaison des traitements pharmacologiques avec la TCC, la symptomatologie persiste chez environ un tiers des patients avec une répercussion fonctionnelle importante [28].

\section{Traitement neurochirurgical (stimulation cérébrale profonde)}

L'identification d'un réseau cortico-sous-cortical dysfonctionnel a incité à envisager des techniques de modulation de l'activité cérébrale pour le traitement des TOC ne répondant pas aux traitements usuels. Si les travaux en stimulation magnétique transcrânienne (TMS) sont encore très préliminaires, la stimulation cérébrale profonde (SCP) apparaît désormais comme une possibilité thérapeutique dans le TOC sévère et résistant (Figure 3).

Jusqu'à présent, différentes cibles ont été essayées: la capsule interne [35], héritage de l'expérience des capsulotomies; le noyau accumbens (NAc) [36], cible pertinente par sa localisation anatomique en considérant l'implication d'un dysfonctionnement du système de récompense dans le TOC; la tête du noyau caudé [37]; et le noyau sous-thalamique (NST) [38]. Le NST est une cible couramment utilisée dans la SCP pour la maladie de Parkinson et a été proposée pour le TOC avec succès [38], à la suite d'une observation de l'amélioration d'obsessions et de compulsions chez des patients parkinsoniens stimulés [39].

Les premiers résultats cliniques d'amélioration des TOC sévères avec la SCP ont suscité un certain nombre de travaux précliniques visant à reproduire les comportements compulsifs chez l'animal et à étudier les effets de la SCP dans ces modèles. Ainsi, on a pu reproduire l'effet de la stimulation à haute fréquence du NST et du NAc $[15,40]$. Chez le primate, un comportement stéréotypé induit par l'injection de bicuculline dans la partie limbique du pallidum externe a également pu être contrôlé par une SCP du NST [41]. Actuellement, les programmes de recherche visent, d'une part, à produire des modèles plus proches de la symptomatologie et, d'autre part, à agir de façon plus spécifique sur des réseaux et des populations neuronales identifiés, en recourant notamment aux techniques d’optogénétique.

\section{(Re)penser le TOC}

Ces dernières années, l'intégration de la sémiologie dimensionnelle, de la neurobiologie, de la génétique et des approches phénoménologiques a entraîné la restructuration nosographique du TOC avec la création d'un groupe dénommé obsessive-compulsive and related disorders proposée par différents auteurs pour le DSM-V et CIM-11 (classification internationale des maladies-11), avec pour objectif l'inclusion du TOC parmi des pathologies comme la trichotillomanie, le syndrome de Gilles de la Tourette ou le trouble de dysmorphie corporelle. L'hétérogénéité du TOC avec les différentes formes d'obsessions et de compulsions constitue un facteur de limitation de puissance pour la recherche qui peut être partiellement corrigée par une approche factorielle à quatre dimensions (symétrie, pensées interdites, lavage, accumulation) $[4,42]$. Cette approche permettrait une meilleure cohérence entre la clinique et les résultats en imagerie cérébrale, ainsi que de la génétique et des thérapeutiques [5, 42, 44]. Au final, l'hétérogénéité du TOC évoque de multiples chevauchements de différents syndromes, constituant des ensembles symptomatiques avec leur propre spécificité, clinique et/ou évolutive. Une meilleure compréhension de ces différences, actuellement réduites dans une même entité nosologique, est souhaitable, dans la perspective du développement d'une thérapeutique personnalisée pour le mieux-être des malades [45-47]. $\diamond$ 


\section{SUMMARY}

\section{The obsessive compulsive disorder}

The obsessive compulsive disorder (OCD) is a frequent disease with a high comorbidity. The usual treatment is a combination of pharmacological and psychotherapeutic treatment. However, $30 \%$ of patients still have persistent and severe symptoms, with an important functional impact. These last years, the integration of the new neuroanatomical, neurochemical, neuropsychological, genetic and phenomenological data, allows a better understanding of the physiopathology and the development of new treatments for OCD, as neuromodulation for the severe and refractory cases. $\diamond$

\section{LIENS D'INTÉRÊT}

Les auteurs déclarent n'avoir aucun lien d'intérêt concernant les données publiées dans cet article.

\section{RÉFÉRENCES}

1. Stein DJ. Obsessive-compulsive disorder. Lancet $2002 ; 360: 397-405$.

2. Kessler RC, Berglund P, Demler 0 , et al. Lifetime prevalence and age-of-onset distributions of DSM-IV disorders in the National comorbidity survey replication. Arch Gen Psychiatry 2005; 62 : 593-602.

3. Abramowitz JS, Taylor S, McKay D. Obsessive-compulsive disorder. Lancet 2009 ; $374: 491-9$.

4. Taylor $S$. Early versus late onset obsessive-compulsive disorder: evidence for distinct subtypes. Clin Psychol Rev $2011 ; 31$ : 1083-100.

5. Katerberg H, Delucchi KL, Stewart SE, et al. Symptom dimensions in OCD: item-level factor analysis and heritability estimates. Behav Genet $2010 ; 40: 505-17$

6. Weissman MM, Bland RC, Canino GJ, et al. The cross national epidemiology of obsessive compulsive disorder. The Cross national collaborative group. J Clin Psychiatry 1994 ; 55 (suppl) : 5-10.

7. Pallanti S. Transcultural observations of obsessive-compulsive disorder. Am J Psychiatry 2008 ; $165: 169-70$.

8. Pauls DL. The genetics of obsessive-compulsive disorder: a review. Dialogues Clin Neurosci 2010 $12: 149-63$.

9. Welch JM, Lu J, Rodriguiz RM, et al. Cortico-striatal synaptic defects and OCD-like behaviours in Sapap3-mutant mice. Nature $2007 ; 448: 894-900$.

10. Nestadt G, Grados M, Samuels JF. Genetics of obsessive-compulsive disorder. Psychiatr Clin North Am 2010; $33: 141-58$.

11. Rotge Jy, Aouizerate B, Tignol J, et al. The glutamate-based genetic immune hypothesis in obsessive-compulsive disorder. An integrative approach from genes to symptoms. Neuroscience $2010 ; 165: 408-17$

12. De Leeuw AS, Westenberg HGM. Hypersensitivity of 5-HT2 receptors in OCD patients. J Psychiatr Res $2008 ; 42: 894-901$.

13. Chakrabarty K, Bhattacharyya S, Christopher R, et al. Glutamatergic dysfunction in OCD. Neuropsychopharmacology $2005 ; 30: 1735-40$.

14. MacMaster FP, O'Neill J, Rosenberg DR. Brain imaging in pediatric obsessive-compulsive disorder.J Am Acad Child Adolesc Psychiatry $2008 ; 47$ : 1262-72.

15. Haynes WIA, Mallet L. High-frequency stimulation of deep brain structures in obsessivecompulsive disorder: the search for a valid circuit. Eur J Neurosci $2010 ; 32$ : 1118-27.

16. Taj MJRJ, Viswanath B, Purushottam M, et al. DRD4 gene and obsessive compulsive disorder: Do symptom dimensions have specific genetic correlates? Prog Neuropsychopharmacol Biol Psychiatry $2013 ; 41: 18-23$.

17. Aouizerate B, Guehl D, Cuny $\varepsilon$, et al. Pathophysiology of obsessive-compulsive disorder: a necessary link between phenomenology, neuropsychology, imagery and physiology. Prog Neurobiol $2004 ; 72: 195-221$.

18. Murphy TK, Storch $E A$, Lewin $A B$, et al. Clinical factors associated with pediatric autoimmune neuropsychiatric disorders associated with streptococcal infections. J Pediatr $2012 ; 160: 314-9$

19. Brilot F, Merheb V, Ding A, et al. Antibody binding to neuronal surface in Sydenham chorea, but not in PANDAS or Tourette syndrome. Neurology $2011 ; 76: 1508-13$.

20. Rotge JY, GuehI D, Dilharreguy B, et al. Meta-analysis of brain volume changes in obsessivecompulsive disorder. Biol Psychiatry $2009 ; 65: 75-83$

21. Szeszko PR, Christian C, Macmaster F, et al. Gray matter structural alterations in psychotropic drug-naive pediatric obsessive-compulsive disorder: an optimized voxel-based morphometry study. Am J Psychiatry $2008 ; 165: 1299-307$.

22. Whiteside SP, Port JD, Abramowitz JS. A meta-analysis of functional neuroimaging in obsessivecompulsive disorder. Psychiatry Res $2004 ; 132: 69-79$.
23. Rotge JY, Langbour N, Jaafari N, et al. Anatomical alterations and symptomrelated functional activity in obsessive-compulsive disorder are correlated in the lateral orbitofrontal cortex. Biol Psychiatry $2010 ; 67$ : e37-8.

24. Zarei M, Mataix-Cols D, Heyman I, et al. Changes in gray matter volume and white matter microstructure in adolescents with obsessive-compulsive disorder. Biol Psychiatry $2011 ; 70$ : 1083-90.

25. Worbe Y, Baup N, Grabli D, et al. Behavioral and movement disorders induced by local inhibitory dysfunction in primate striatum. Cerebral Cortex 2009; 19 : 1844-56.

26. Grabli D. Behavioural disorders induced by external globus pallidus dysfunction in primates. I. Behavioural study. Brain 2004 ; $127: 2039-54$.

27. Karachi C, Grabli D, Baup N, et al. Dysfunction of the subthalamic nucleus induces behavioral and movement disorders in monkeys. Mov Disord 2009; 24 : 1183-92.

28. Pallanti S, Quercioli L. Treatment-refractory obsessive-compulsive disorder: methodological issues, operational definitions and therapeutic lines. Prog Neuropsychopharmacol Biol Psychiatry 2006; 30:400-12

29. Bloch MH, Landeros-Weisenberger A, Kelmendi B, et al. A systematic review: antipsychotic augmentation with treatment refractory obsessivecompulsive disorder. Mol Psychiatry 2006 ; 11 : 622-32.

30. Komossa K, Depping AM, Meyer M, et al. Second-generation antipsychotics for obsessive compulsive disorder. Cochrane Database Syst Rev 2010 CD008141.

31. Abramowitz JS. The psychological treatment of obsessive-compulsive disorder. Can J Psychiatry 2006 ; $51:$ 407-16.

32. Storch EA, Mariaskin A, Murphy TK. Psychotherapy for obsessive-compulsive disorder. Curr Psychiatry Rep 2009; 11 : 296-301.

33. Foa $\varepsilon B$, Franklin ME, Moser J. Context in the clinic: how well do cognitivebehavioral therapies and medications work in combination? Biol Psychiatry $2002 ; 52$ : 987-97

34. Storch EA, Murphy TK, Goodman WK, et al. A Preliminary study of $D$-cycloserine augmentation of cognitive-behavioral therapy in pediatric obsessive-compulsive disorder. Biol Psychiatry 2010 ; 68 : 1073-6.

35. Nuttin B, Cosyns $P$, Demeulemeester $H$, et al. Electrical stimulation in anterior limbs of internal capsules in patients with obsessive-compulsive disorder. Lancet $1999 ; 354: 1526$

36. Denys $D$, Mantione M, Figee $M$, et al. Deep brain stimulation of the nucleus accumbens for treatment-refractory obsessive-compulsive disorder. Arch Gen Psychiatry 2010 ; 67 : 1061-8.

37. Aouizerate B, Cuny $\varepsilon$, Martin-Guehl C, et al. Deep brain stimulation of the ventral caudate nucleus in the treatment of obsessive-compulsive disorder and major depression. Case report. J Neurosurg 2004 ; 101 : 682-6.

38. Mallet L, Polosan M, Jaafari N, et al. Subthalamic nucleus stimulation in severe obsessive-compulsive disorder. N Engl / Med 2008 ; 359 : 2121-34.

39. Mallet L, Mesnage V, Houeto JL, et al. Compulsions, Parkinson's disease, and stimulation. Lancet $2002 ; 360: 1302-4$

40. Mundt A, Klein J, Joel D, et al. High-frequency stimulation of the nucleus accumbens core and shell reduces quinpirole-induced compulsive checking in rats. Eur J Neurosci $2009 ; 29: 2401-12$

41. Baup N, Grabli D, Karachi C, et al. High-frequency stimulation of the anterior subthalamic nucleus reduces stereotyped behaviors in primates. Neurosci $2008 ; 28: 8785-8$.

42. Bloch MH, Landeros-Weisenberger A, Rosario MC, et al. Meta-analysis of the symptom structure of obsessive-compulsive disorder. Am J Psychiatry 2008; $165: 1532-42$.

43. Mataix-Cols D, Rosario-Campos MCD, Leckman JF. A multidimensional model of obsessive-compulsive disorder. Am J Psychiatry $2005 ; 162: 228-38$.

44. Harrison BJ, Pujol J, Cardoner N, et al. Brain corticostriatal systems and the major clinical symptom dimensions of obsessive-compulsive disorder. Biol Psychiatry $2013 ; 73$ : 321-8.

45. Aouizerate B, Martin-Guehl C, Cuny $\varepsilon$, et al. Stimulation cérébrale profonde du striatum ventral dans le traitement du trouble obsessionnel-compulsif avec dépression majeure. Med Sci (Paris) 2005 ; 21 : 811-3.

46. Epelbaum J. Pour les tics et tocs, serait-ce SLITRK1 ? Med Sci (Paris) 2005 $21: 1117$.

47. Jupille J. Le trouble déficitaire de l'attention avec hyperactivité. Med Sci (Paris) $2011 ; 27: 318-22$.

\section{TIRÉS À PART}

L. Mallet 